\title{
Eye Can See What You Want: Posterior Intraparietal Sulcus Encodes the Object of an Actor's Gaze
}

\author{
Richard Ramsey ${ }^{1}$, Emily S. Cross ${ }^{2}$, and Antonia F. de C. Hamilton ${ }^{1}$
}

\begin{abstract}
In a social setting, seeing Sally look at a clock means something different to seeing her gaze longingly at a slice of chocolate cake. In both cases, her eyes and face might be turned rightward, but the information conveyed is markedly different, depending on the object of her gaze. Numerous studies have examined brain systems underlying the perception of gaze direction, but less is known about the neural basis of perceiving gaze shifts to specific objects. During fMRI, participants observed an actor look toward one of two objects, each occupying a distinct location. Video stimuli were sequenced to obtain repetition suppression (RS) for object identity, independent of spatial location. In a control condition, a spotlight highlighted one of the objects, but no actor was present. Observation of the human actor's gaze compared with the spotlight engaged frontal,
\end{abstract}

\section{INTRODUCTION}

Attending to other people's gaze can provide information about their mental states, such as their interests and goals (Emery, 2000). For example, if your dinner companion's gaze rests upon a wine bottle, this might indicate that he would like a drink. Recently, an increasing interest in the brain systems involved in gaze-based social interactions has distinguished regions selective to gaze direction (Nummenmaa \& Calder, 2009). Here we test which brain regions are sensitive to the object, rather than the spatial location, that becomes the focus of an observed person's gaze.

A broad network of brain regions has been associated with the perception of eye gaze (Nummenmaa \& Calder, 2009; Grosbras, Laird, \& Paus, 2005). Within this network, the STS and intraparietal sulcus (IPS) have been associated with different functional processes during gaze perception (Nummenmaa, Passamonti, Rowe, Engell, \& Calder, 2010; Hoffman \& Haxby, 2000). Anterior and posterior segments of STS (aSTS and pSTS, respectively) are associated with processing gaze direction (Calder et al., 2007; Pelphrey, Viola, \& McCarthy, 2004; Hooker et al., 2003; Pelphrey, Singerman, Allison, \& McCarthy, 2003; Puce, Allison, Bentin, Gore, \& McCarthy, 1998; Wicker, Michel, Henaff, \& Decety, 1998; Perrett et al., 1985). In contrast, IPS has been shown to respond specifically to

${ }^{1}$ University of Nottingham, ${ }^{2}$ Radboud University Nijmegen parietal, and temporal cortices, consistent with a broad action observation network. RS for gazed object in the human condition was found in posterior intraparietal sulcus (pIPS). RS for highlighted object in the spotlight condition was found in middle occipital, inferior temporal, medial fusiform gyri, and superior parietal lobule. These results suggest that human pIPS is specifically sensitive to the type object that an observed actor looks at (tool vs. food), irrespective of the observed actor's gaze location (left vs. right). A general attention or lower-level object feature processing mechanism cannot account for the findings because a very different response pattern was seen in the spotlight control condition. Our results suggest that, in addition to spatial orienting, human pIPS has an important role in object-centered social orienting.

attention-shifting aspects of gaze perception (Materna, Dicke, \& Thier, 2008; Hietanen, Nummenmaa, Nyman, Parkkola, \& Hämäläinen, 2006) and the observer's tendency to shift gaze and attention in the same direction as the observed individual (Driver et al., 1999; Langton \& Bruce, 1999; Friesen \& Kingstone, 1998). This proposal is compatible with existing theories of attention that suggest IPS is part of a dorsal fronto-parietal attention network that coordinates stimulus-response selection (Corbetta, Patel, \& Shulman, 2008; Corbetta \& Shulman, 2002).

Recent single-unit recordings in rhesus macaque monkeys provide more detail for the role of STS and IPS in social gaze perception. Monkeys provide a suitable test case for theories of human gaze perception because they follow the attention of others (Emery, Lorincz, Perrett, Oram, \& Baker, 1997) and gaze-follow in a similar way to humans (Deaner \& Platt, 2003). Research in this vein has revealed that neurons within STS encode observed gaze direction (Perrett et al., 1985). Moreover, neurons in lateral intraparietal area (LIP), a brain region known as the parietal eye field by some researchers (Andersen, Brotchie, \& Mazzoni, 1992), respond when a monkey looks toward a region in space and also when a monkey observes another monkey perform the same task (Shepherd, Klein, Deaner, \& Platt, 2009). This LIP finding is consistent with recent evidence showing that LIP neurons compute a saliency or priority map, a metric of where to look (Bisley \& Goldberg, 2010; Klein, Shepherd, \& Platt, 2009; Itti \& Koch, 2000). 
Such findings suggest that, during gaze perception, LIP neurons orient attention to the location of important stimuli in the environment. Together, these studies support the hypothesis that, within the primate brain, gaze direction is processed in STS, whereas attention-orienting following observed gaze is encoded within posterior parietal brain regions.

In human social interactions, gaze shifts can be deictic; that is, they can "point-out" the spatial location of relevant items in the environment, such as objects and conspecifics (Shepherd, 2010). Deictic gaze shifts are typically preceded by direct gaze to signal communicative intent (Schilbach et al., 2006; Kampe, Frith, \& Frith, 2003) and result in joint attention. Following Emery's (2000) definition, joint attention involves two individuals attending to the same specific feature of the environment on the basis of one individual responding to the cues of the other individual. In contrast, gaze following may be simpler and only involve orienting to a region in space, such as the left or right hemifield, rather than a specific feature of the environment.

Observation of deictic gaze consistently engages pSTS, posterior parietal cortex, and medial pFC (mPFC; Redcay et al., 2010; Schilbach et al., 2010; Materna et al., 2008; Bristow, Rees, \& Frith, 2007; Williams, Waiter, Perra, Perrett, \& Whiten, 2005). mPFC activity during deictic gaze is believed to reflect the communication of socially relevant information regarding the object at the focus of joint attention (Saxe, 2006). Furthermore, when target objects of deictic gaze are graspable, then the inferior frontal gyrus (IFG) and inferior parietal lobule (IPL) respond in addition to pSTS (Becchio, Bertone, \& Castiello, 2008; Pierno, Becchio, Tubaldi, Turella, \& Castiello, 2008; Pierno et al., 2006). IFG and IPL respond when performing and observing hand actions (Grèzes \& Decety, 2001), which suggests that observing gaze directed toward graspable objects produces a representation of grasp in the observer (Pierno et al., 2006).

In summary, a broad network of brain regions respond during gaze perception (Nummenmaa \& Calder, 2009; Grosbras et al., 2005), and many studies have investigated and discussed the role that STS plays in this process (Birmingham \& Kingstone, 2009). To date, less is known about the functional role played by the wider gaze network, including fronto-parietal attention systems (Nummenmaa et al., 2010). For instance, although posterior parietal cortex, specifically monkey LIP and human posterior IPS (pIPS), is sensitive to shifts in spatial attention during gaze perception, it is not known if posterior parietal or any other component of the gaze perception network is sensitive to the type of object that becomes the focus of an observed individual's gaze.

Here we aimed to go beyond previous work that focused on the spatial nature of gaze perception and consider how deictic gaze in humans is linked to specific, meaningful objects. We use a repetition suppression (RS) design during fMRI, which has proved successful in previous studies of action perception (Ramsey \& Hamilton, 2010a, 2010b, in press; Hamilton \& Grafton, 2006, 2007). $\mathrm{RS}$ is grounded in the principle that the presentation of a repeated stimulus feature will result in a reduced BOLD response in brain regions that encode that stimulus feature (Grill-Spector \& Malach, 2001). Previous work has demonstrated that anterior IPS (aIPS) is sensitive to the type of object (tool vs. food) that is observed being grasped by a hand (Hamilton \& Grafton, 2006, 2007). We used a similar paradigm to study how the human brain responds to a gazed-at object in a deictic attention situation. In particular, we aimed to test the contributions of STS and pIPS to encoding the identity of the object in a deictic gaze situation. Furthermore, we aimed to contrast the response in STS and pIPS with responses in ventral temporal areas, which have been associated with processing object properties, such as color (Simmons et al., 2007; Beauchamp, Haxby, Jennings, \& DeYoe, 1999; Chao \& Martin, 1999) and category (Haxby et al., 1999, 2001; Ishai, Ungerleider, Martin, \& Haxby, 2000; Chao, Haxby, \& Martin, 1999; Grill-Spector et al., 1999; Ishai, Ungerleider, Martin, Schouten, \& Haxby, 1999). If object properties such as color and category are encoded, rather than a process specifically related to deictic gaze perception, we would expect responses in ventral temporal regions (Martin, 2007).

\section{METHODS}

Twenty-eight participants ( 14 men, mean age $=25.9$ years $)$ gave informed consent. Participants watched movie clips in two different conditions: human and spotlight. In the human condition, a female actor sat at a table with two objects. She started each trial with her head facing downward. Then, she looked directly toward the camera (direct gaze) before directing her gaze toward one object (Figure 1A). Direct gaze was included to provide a social cue that would engage participants' attention (Senju \& Hasegawa, 2005). In the spotlight condition, a lightly colored (transparent) circle appeared centrally before moving and remaining over one object (Figure 1B). Consistent with previous research (Ramsey \& Hamilton, 2010a, 2010b; Hamilton \& Grafton, 2006, 2007), the two objects were matched in terms of their size and shape but comprised one food (e.g., apple) and one nonfood item (e.g., camera) to distinguish object category. Three object pairs were used across three functional runs: apple-camera, banana-screwdriver, and orange-wooden blocks. In each functional run, eight sets of videos alternated between human and spotlight conditions. Different object pairs were used within the same functional run for human and spotlight conditions to avoid any unwanted suppression between sets. For example, in one functional run, sets of videos alternated between apple-camera (human condition) and bananascrewdriver (spotlight condition). Movies were $2.67 \mathrm{sec}$ long and 640 pixels wide by 480 pixels high and separated by a black screen for $0.4 \mathrm{sec}$. All stimuli were presented with Cogent running under Matlab 6.5. 


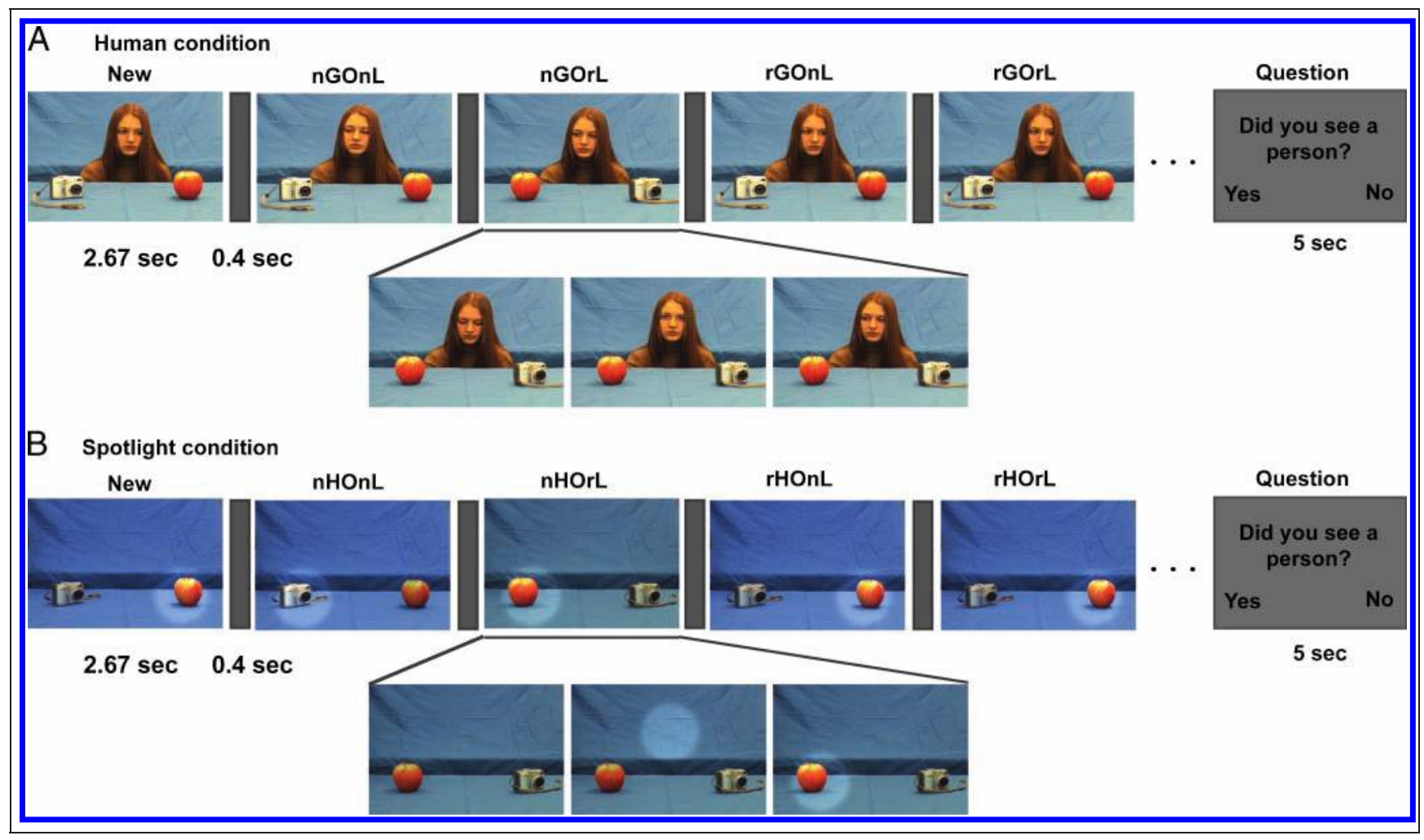

Figure 1. Stimulus sequencing. (A) In the human condition, a female actor gazed straightforward and then toward one of two objects (lower panel). (B) In the spotlight condition, a transparent spotlight appeared centrally before moving and highlighting one of two objects (bottom). Target objects were always one food item and one nonfood item. In the example shown, an apple and a camera are shown in each clip. Sequences of nine movies always started with a "new" clip followed by eight clips depicting a novel (n) or repeated (r) gazed object (GO), highlighted object (HO), or location (L). Novelty was defined relative to the previous movie in a one-back design. Following a sequence, participants answered a question to maintain alertness.

Movies were sequenced to obtain one-back RS (Figure 1). An RS design was used because of the potential to demonstrate that separate cell populations are tuned to different stimulus categories, even if they are intermixed within the same imaging voxel or brain region (Naccache \& Dehaene, 2001). Consequently, compared with conventional subtraction fMRI designs, RS can examine what populations of neurons are coding within a brain area and not just between brain areas. Sequences of nine movies always started with a "new" clip followed by eight clips depicting a novel (n) or repeated $(\mathrm{r})$ object $(\mathrm{O})$ or location $(\mathrm{L})$. Following a sequence, participants answered a question about the content of the last movie they had just observed to maintain alertness. Each participant completed 192 RS trials, which evenly filled a 2 (Object: novel and repeated) $\times 2$ (Location: novel and repeated) $\times 2$ (Cue: human and spotlight) factorial design. Scanning was performed in a $3 \mathrm{~T}$ Phillips Achieva scanner using an eight-channel-phased array head coil with 40 slices per repetition time (3-mm thickness); repetition time $=2500 \mathrm{msec}$, echo time $=40 \mathrm{msec}$, flip angle $=80^{\circ}$, field of view $=19.2 \mathrm{~cm}$, matrix $=64 \times 64$. One hundred twenty brain images were stored on each of three functional runs. Data were realigned, unwarped, corrected for slice timing, normalized to the Montreal Neurological Institute template with a resolution of $3 \times 3 \times 3 \mathrm{~mm}$, and spa- tially smoothed (8 mm) using the SPM8 software. A design matrix was fitted for each participant with regressors for each movie type (nOnL, nOrL, rOnL, rOrL, new, and question) separately for human and spotlight conditions. Each trial was modeled as a boxcar with the duration of that movie convolved with the standard hemodynamic response function.

The first analysis examined human $>$ spotlight and spotlight $>$ human, across all movies. Then RS for gazed object was examined by contrasting novel gazed object $>$ repeated gazed object for the human videos only. RS for gazed object does not involve differences in "communicative intent" (Kuzmanovic et al., 2009; Schilbach et al., 2006; Kampe et al., 2003), because such communicative information is balanced between novel and repeated trials. That is, on every trial the actor directs her gaze toward the participant before gazing toward one object. Instead, the only difference between novel and repeated trials is the sequence of novel and repeated gazed-at object types. In addition to a whole-brain analysis, a small volume correction was applied using two a priori ROIs. First, a bilateral pIPS ROI was focused on coordinates taken from Konen and Kastner's (2008) retinotopic mapping of IPS. Two sections of pIPS, IPS1 and IPS2, show anatomical and functional equivalence to macaque 
LIP and are believed to be the human homologue of this region (Silver \& Kastner, 2009). Ten-millimeter spheres were centered on the mean-averaged coordinates for IPS1 and IPS2 (left: $-25,-76,43$ ), which showed consistency with other retinotopic maps of IPS (Hagler, Riecke, \& Sereno, 2007; Levy, Schluppeck, Heeger, \& Glimcher, 2007; Schluppeck, Glimcher, \& Heeger, 2005; Sereno, Pitzalis, \& Martinez, 2001). Second, a bilateral pSTS ROI was focused on coordinates (left: $-48,-56,16)$ taken from a gaze perception meta-analyses (Grosbras et al., 2005). The main effect of RS for gazed location (novel > repeated) and the interaction between gazed object and gazed location were also calculated for human videos only. In Table 1, the results of the whole-brain (top section) and ROI analyses (bottom section) are reported. RS for highlighted object was examined by contrasting novel highlighted object $>$ repeated highlighted object for spotlight videos only. In addition, the main effect of RS for highlighted location (novel $>$ repeated) and the interaction between highlighted object and highlighted location were calculated for the spotlight videos. Correction for multiple comparisons was performed at the cluster level (Friston, Worsley, Frackowiak, Mazziotta, \& Evans, 1994), using a voxel level threshold of $p<.005$ and 10 voxels and an family-wise error (FWE) cluster level correction of $p<$ .05 . Brain regions were localized using a human brain atlas (Duvernoy, 1999) in combination with an on-line search tool (http://sumsdb.wustl.edu/sums/).

\section{RESULTS}

The main effect of human compared with spotlight stimuli (human $>$ spotlight) revealed that bilateral STS, right IFG, right IPL, and right amygdala showed greater activity in the human condition compared with the spotlight condition (Supplementary Figure 1 and Supplementary Table 1). The inverse contrast revealed that bilateral medial fusiform and left superior parietal lobule (SPL) showed greater activity in the spotlight condition compared with the human condition (Supplementary Figure 2 and Supplementary Table 2).

Within the human condition, the whole-brain analysis of RS for gazed object yielded one cluster located in left pIPS, extending to the SPL and precuneus (Table 1, top section). We also performed two ROI analyses that were based on our a priori hypotheses, which confirmed this pattern of activity in pIPS. The bilateral pIPS small volume revealed that the left pIPS survived correction for multiple

Table 1. Brain Regions Showing RS for Gazed Object and RS for Gazed Location in the Human Condition

\begin{tabular}{|c|c|c|c|c|c|c|}
\hline \multirow[b]{2}{*}{ Region } & \multirow[b]{2}{*}{ Number of Voxels } & \multirow[b]{2}{*}{$t$} & \multirow{2}{*}{$\begin{array}{c}p \text { Cluster Corrected } \\
\text { (FWE) }\end{array}$} & \multicolumn{3}{|c|}{$\begin{array}{l}\text { Montreal Neurological } \\
\text { Institute Coordinates }\end{array}$} \\
\hline & & & & $x$ & $y$ & $z$ \\
\hline \multicolumn{7}{|l|}{ Whole-brain Analysis } \\
\hline \multicolumn{7}{|l|}{ RS for gazed object } \\
\hline \multirow{3}{*}{$\begin{array}{l}\text { Left pIPS extending into SPL } \\
\text { and precuneus }\end{array}$} & \multirow[t]{3}{*}{100} & \multirow[t]{3}{*}{3.75} & \multirow[t]{3}{*}{.161} & -24 & -76 & 43 \\
\hline & & & & -12 & -79 & 43 \\
\hline & & & & -12 & -67 & 46 \\
\hline \multicolumn{7}{|l|}{ RS for gazed location } \\
\hline \multirow[t]{2}{*}{ Left caudate (medial wall) } & \multirow[t]{2}{*}{19} & \multirow[t]{2}{*}{4.43} & \multirow[t]{2}{*}{.993} & -9 & -1 & 22 \\
\hline & & & & -21 & 2 & 22 \\
\hline Right posterior cingulate & 26 & 3.95 & .960 & 9 & -34 & 16 \\
\hline Left thalamus & 11 & 3.65 & 1.000 & -3 & -19 & -5 \\
\hline
\end{tabular}

ROI Analysis: RS for Gazed Object

Small volume correction 1: bilateral pIPS

Left pIPS

52
$<.01$

$\begin{array}{lll}-24 & -76 & 43 \\ -15 & -79 & 46\end{array}$

Small volume correction 2: bilateral pSTS

No brain regions

For whole-brain and ROI analyses, only regions surviving a voxel level threshold of $p<.005$ and 10 voxels are reported. Subpeaks of more than $8 \mathrm{~mm}$ from the main peak in each cluster are listed.

Bold indicates regions that survived the cluster-corrected (FWE) threshold at $p<.05$. 
Figure 2. Left pIPS shows RS for gazed object in the human condition. Significant suppression was seen for repeated gazed object (white columns) compared with novel gazed object (blue columns) in pIPS. Clusteraverage parameter estimates (SPM betas) are shown in Plot 1 A similar RS pattern was not observed for highlighted object in pIPS (Plot 2). Abbreviations: $\mathrm{n}=$ novel, $\mathrm{r}=$ repeated, $\mathrm{GO}=$ gazed object, $\mathrm{HO}=$ highlighted object, $\mathrm{L}=$ location. Results are from the whole-brain analysis.

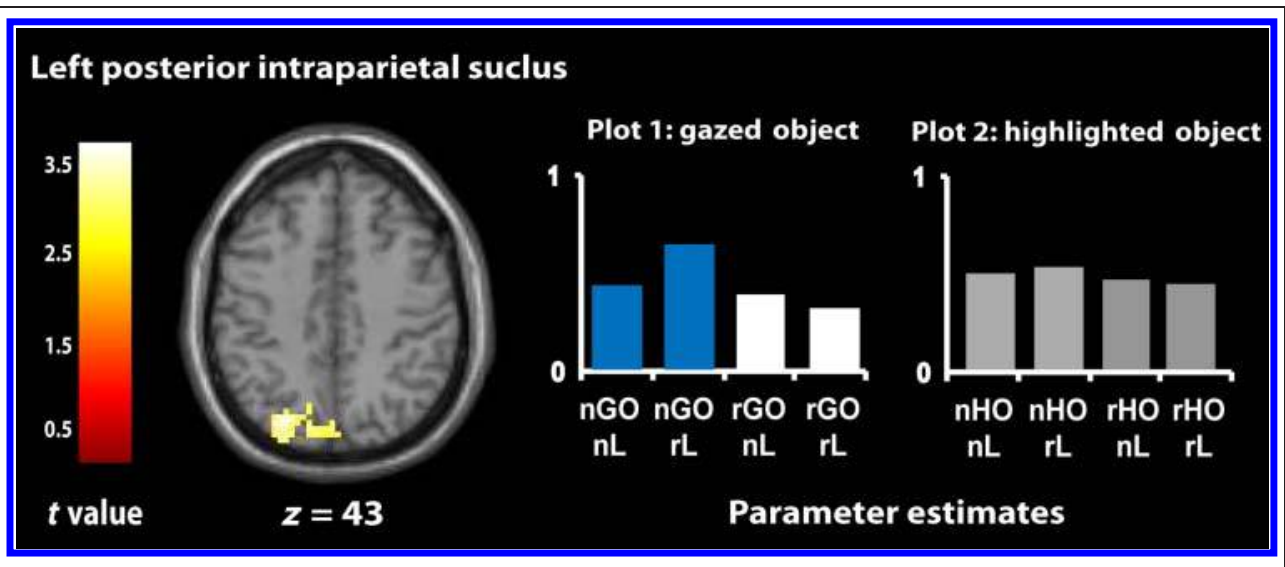

comparisons $(p<.05$, FWE). In contrast, the bilateral pSTS small volume revealed no such pattern of activity, even at uncorrected thresholds (Table 1, bottom section). In Figure 2, the pattern of response in the pIPS cluster is depicted with parameter estimate plots showing that irrespective of gazed location, the response to observed gaze directed at a novel object was suppressed when the same object was gazed at for a second time. No brain regions showed RS for gazed location at the corrected threshold, and only three regions-caudate, thalamus, and posterior cingulatemet the uncorrected threshold (Table 1). There were no significant interactions between gazed object and gazed location.

In the spotlight condition, RS for highlighted object was seen bilaterally in middle occipital gyrus, medial fusiform gyrus, inferior temporal gyrus, and SPL. Figure 3 illustrates the pattern of response in these brain regions with parameter estimate plots showing that, irrespective of highlighted location, the response to a novel highlighted object was suppressed when the same object was highlighted for a second time. No brain regions showed RS for highlighted location at the corrected threshold, and only two regionscaudate and thalamus - met the uncorrected threshold (Table 2). There were no significant interactions between highlighted object and highlighted location.

\section{DISCUSSION}

Our findings demonstrate that observing someone repeatedly gaze at the same object suppresses the BOLD

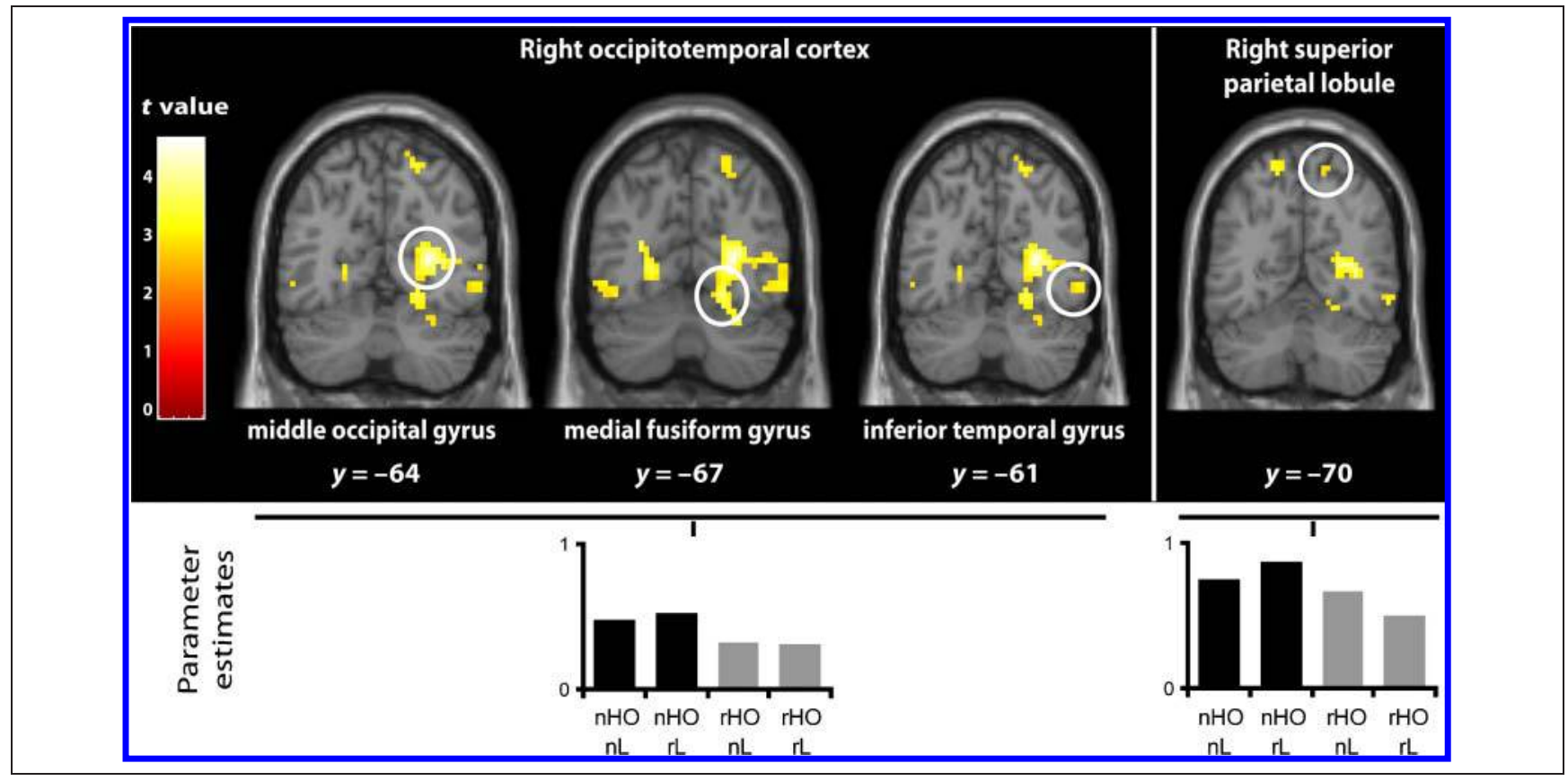

Figure 3. Brain regions showing RS for highlighted object in the spotlight condition. Significant suppression was seen for repeated highlighted object (gray columns) compared with novel highlighted object (black columns) in bilateral middle occipital, medial fusiform, inferior temporal gyrus, and SPL (images are centered on coordinates for right hemisphere cluster peaks and subpeaks). Cluster-average parameter estimates (SPM betas) are plotted for each cluster. Abbreviations: $\mathrm{n}=$ novel, $\mathrm{r}=$ repeated, $\mathrm{HO}=$ highlighted object, $\mathrm{L}=$ location. In clusters with anatomically distinct subpeaks, each subpeak is visualized and circled. 
Table 2. Brain Regions Showing RS for Highlighted Object and RS for Highlighted Location in the Spotlight Condition

\begin{tabular}{|c|c|c|c|c|c|c|}
\hline \multirow[b]{2}{*}{ Region } & \multirow[b]{2}{*}{ Number of Voxels } & \multirow[b]{2}{*}{$t$} & \multirow{2}{*}{$\begin{array}{c}p \text { Cluster Corrected } \\
\text { (FWE) }\end{array}$} & \multicolumn{3}{|c|}{$\begin{array}{l}\text { Montreal Neurological } \\
\text { Institute Coordinates }\end{array}$} \\
\hline & & & & $x$ & $y$ & $z$ \\
\hline \multicolumn{7}{|l|}{ RS Highlighted Object } \\
\hline \multirow{3}{*}{$\begin{array}{l}\text { Right middle occipital gyrus extending } \\
\text { into inferior temporal and medial } \\
\text { fusiform gyri }\end{array}$} & 453 & 4.55 & $<.001$ & 27 & -64 & 7 \\
\hline & & & & 36 & -61 & 7 \\
\hline & & & & 21 & -67 & -11 \\
\hline \multirow[t]{3}{*}{ Left posterior insula } & 45 & 4.44 & .858 & -33 & -10 & 13 \\
\hline & & & & -39 & -25 & 19 \\
\hline & & & & -33 & -19 & 10 \\
\hline \multirow[t]{2}{*}{ Left middle occipital gyrus } & 55 & 3.66 & .757 & -24 & -70 & 4 \\
\hline & & & & -27 & -73 & 16 \\
\hline Left SPL & 16 & 3.42 & .998 & -15 & -58 & 64 \\
\hline Left lateral middle occipital gyrus & 20 & 3.31 & .993 & -39 & -85 & 16 \\
\hline \multirow[t]{2}{*}{ Left medial fusiform gyrus } & 28 & 3.28 & .973 & -18 & -76 & -14 \\
\hline & & & & -24 & -82 & -8 \\
\hline \multirow[t]{3}{*}{ Right SPL } & 21 & 3.19 & .992 & 24 & -70 & 58 \\
\hline & & & & 24 & -70 & 49 \\
\hline & & & & 15 & -61 & 64 \\
\hline \multirow[t]{2}{*}{ Left inferior temporal gyrus } & 14 & 3.12 & .999 & -51 & -67 & -11 \\
\hline & & & & -42 & -67 & -11 \\
\hline \multirow[t]{3}{*}{ Left medial occipital } & 25 & 3.06 & .983 & -6 & -76 & 7 \\
\hline & & & & -15 & -85 & 4 \\
\hline & & & & 6 & -79 & 13 \\
\hline \multicolumn{7}{|l|}{ RS Highlighted Location } \\
\hline \multirow[t]{2}{*}{ Left caudate extending into thalamus } & 32 & 4.97 & .943 & -24 & -13 & 19 \\
\hline & & & & -21 & -7 & 25 \\
\hline \multirow[t]{2}{*}{ Left caudate tail } & 24 & 3.57 & .983 & -24 & -37 & 13 \\
\hline & & & & -24 & -46 & 7 \\
\hline
\end{tabular}

Only regions surviving a whole-brain voxel level threshold of $p<.005$ and 10 voxels are reported. Subpeaks of more than $8 \mathrm{~mm}$ from the main peak in each cluster are listed.

Bold indicates regions that survived the whole-brain cluster-corrected (FWE) threshold at $p<.05$.

response in pIPS, whereas observing someone gazing at a novel object results in release from suppression in this region. In contrast, when a spotlight highlighted the same sequence of objects, brain regions implicated in a broad object-processing network responded (fusiform gyrus, inferior temporal gyrus, middle occipital gyrus, and SPL). These findings suggest that pIPS is specifically sensitive to object identity in the context of deictic gaze perception.

\section{Gaze Perception and pIPS}

Our study is the first investigation of brain systems sensitive to the identity of gazed-at objects in the context of deictic gaze. We find that left pIPS is the only brain region sensitive to the relationship between observed eye gaze and object identity. Previous fMRI studies of deictic gaze perception associate the pIPS with shifts of spatial attention (Materna et al., 2008; Hietanen et al., 2006) within a 
broader gaze-perception brain network (Nummenmaa \& Calder, 2009; Grosbras et al., 2005). Within this broad network, mPFC has been shown to reflect communicative intent (Kuzmanovic et al., 2009; Schilbach et al., 2006; Kampe et al., 2003), whereas pSTS has been shown to respond to the direction and intentionality of gaze (Nummenmaa \& Calder, 2009; Allison, Puce, \& McCarthy, 2000). In the present study, no other brain region showed the same response as pIPS, including pSTS and mPFC. This finding supports and extends the proposed functional role of the parietal node of the gaze perception network: During deictic gaze perception, pIPS responds to object-centered attention shifts, in addition to spatial attention shifts.

Before discussing the implications of this result, we highlight features of the experimental design that support our interpretation of the result in pIPS and rule out alternative viewpoints. The result cannot reflect sensitivity to observed gaze direction, because the RS for gazed object contrast is not based on a distinction between left and right gaze direction. Instead, it is based on observed gaze toward a particular object (tool vs. food), irrespective of the observed actor's gaze direction (left vs. right). On a related note, the result cannot be accounted for by reorienting of spatial attention (i.e., reorienting to a location in space), because such an explanation is not tied to the identity of the object. Any brain region showing RS for gazed object shows sensitivity to the identity of the gazed-at object independent of the object's spatial location. The result also cannot be explained by object processing alone. If this were the case, the same response should have been observed in the spotlight condition, wherein the same objects were highlighted. Such a pattern was not seen in the spotlight condition even when using a small volume correction for pIPS. Instead, brain regions associated with processing lower-level object features responded in the spotlight condition. We did not control for participants' eye movements in the scanner, but we did perform a secondary behavioral experiment using eye-tracking equipment outside the scanner (Supplementary Figure 3). This demonstrated that the number of saccades and the proportion of time spent looking at the person's face did not differ between novel and repeated gazed object trials. This suggests that eye movement cannot account for the RS for gazed object effect observed in pIPS. For these reasons, we believe that our result reflects sensitivity to the identity of an object that becomes the focus of an observed individual's eye gaze.

Posterior parietal cortex, which includes pIPS, is considered part of the dorsal attention reorienting system, which, together with the FEFs, is involved in both goaldirected and stimulus-driven orienting of attention, with a particular role in selecting targets to attend (Corbetta et al., 2008; Corbetta \& Shulman, 2002). It seems likely that an important evolutionary cue to attention reorientation is the gaze direction of others, as this information could enhance chances of survival (Emery, 2000). Associating the spatial location of attention through eye gaze has been localized to LIP in monkeys and similar regions in humans (Shepherd, 2010). This has been taken as evidence that monkey LIP and human pIPS computes a social saliency or priority map of where to look (Klein et al., 2009; Shepherd et al., 2009). Here we extend this spatial attention proposal by demonstrating that pIPS is sensitive to eye gaze shifts that reorient attention toward object identity, irrespective of spatial location. Thus, we distinguish two types of attention reorienting: spatial and object based. Spatial reorienting consists of reorienting attention to a location in space, whereas object-based reorienting consists of reorienting attention to a particular type of object, irrespective of its spatial location. We suggest that pIPS may compute a saliency map for the object gazed at by another person, which could be of evolutionary importance if one considers the difference between seeing someone look toward food or a predator occupying the same location. In such a case, attention shifts to the same position in space but the different identities of the gazed-at item can convey different meaning, for example, one may wish to approach food but want to avoid a predator.

The result complements recent action observation research that showed aIPS is sensitive to the identity of an object that is seen grasped by a hand (Hamilton \& Grafton, 2006, 2007). Previously, aIPS had been associated with grasp shape (Culham, Cavina-Pratesi, \& Singhal, 2006; Sakata, Taira, Murata, \& Mine, 1995), but this recent work suggests that aIPS is also sensitive to higher-level features of observed actions, such as object goal (Grafton, 2009; Tunik, Rice, Hamilton, \& Grafton, 2007). Here we show a comparable pattern of results in pIPS for eye gaze perception. Previously, monkey LIP and human pIPS have been implicated in controlling and perceiving the direction of eye movements (Silver \& Kastner, 2009; Grosbras et al., 2005; Andersen et al., 1992; Gnadt \& Andersen, 1988). We develop this position by demonstrating that pIPS is sensitive to the identity of an object that is gazed at by someone else. Together, this evidence suggests that aIPS and pIPS may encode distinct elements of perception that are higher in a hierarchical structure of social information processing than just grasp shape and gaze direction, respectively. Future work could investigate this proposal by testing how lower-level features of social perception (e.g., grasp shape and gaze direction) and higher-level features (e.g., object-directed grasping and deictic gaze) interact and subsequently influence responses along the IPS.

Our results do not suggest that only pIPS is involved in gaze perception. We show that pSTS responds robustly to human gaze compared with a no-gaze control, which supports previous work implicating pSTS with numerous functions during gaze perception (Nummenmaa \& Calder, 2009; Grosbras et al., 2005). In addition to pSTS, using the same contrast, we show that IFG and IPL respond. This is consistent with recent findings that suggest observed gaze directed toward graspable objects elicits a representation 
of grasp in the observer (Pierno et al., 2006, 2008). Thus, although we demonstrate that a broad network of brain regions responds during gaze perception, our finding that pIPS alone shows RS for gazed object provides a more subtle extension to the gaze perception literature. The result suggests that pIPS plays a specific role in linking an observed individual's gaze with object identity. A similar result is not observed in pSTS or elsewhere in the brain. These findings further fractionate the gaze perception network, suggesting that distinct nodes may have distinct functional processes (Hoffman \& Haxby, 2000), with posterior parietal cortex encoding object-centered attention shifts. Future work that attempts to demonstrate how these brain regions interact during social gaze perception would be valuable (Nummenmaa et al., 2010).

\section{Nonsocial Attention to Objects}

To rule out the possibility that our findings in the human condition reflect a general attention-reorienting mechanism rather than one specific to social gaze perception, we included a nonsocial condition. In this spotlight condition, no sensitivity to the highlighted object was observed within pIPS, even when applying a small volume correction. Instead, ventral temporal, occipital, and superior parietal cortices responded when a spotlight highlighted the same sequence of objects as used in the human condition. These regions are commonly implicated as components of a distributed neural network that process object properties, such as color (Simmons et al., 2007; Beauchamp et al., 1999; Chao \& Martin, 1999), as well as object categories, such as tools and animals (Haxby et al., 1999, 2001; Ishai et al., 1999, 2000; Chao et al., 1999; Grill-Spector et al., 1999). We interpret the current findings in a manner consistent with these data, suggesting that, in the absence of a human agent, the spotlight did not elicit deictic gaze. Instead, lower-level object properties (e.g., color) or object categories (e.g., tool or food) were encoded.

\section{Gaze Direction}

Contrary to predictions, no portion of STS showed sensitivity to gaze direction (RS for gazed location), even at lenient statistical thresholds; nor did any other brain region at corrected thresholds. Differences in experimental paradigms between studies may account for this negative result. Using a suppression design, one previous fMRI study showed that aSTS and IPL were sensitive to gaze direction (Calder et al., 2007). The type of (repetition) suppression involved was quite different to the one employed by the current study. Calder and colleagues first "adapted" participants to a particular gaze direction (e.g., left) through observing a series of face images gazing left. Subsequently, they tested if a face with a congruent gaze direction (i.e., left) suppressed the BOLD response compared with a face with an incongruent or neutral gaze direction. In contrast, the current experiment did not "adapt" participants to a particular gaze direction, but instead used a "one-back" suppression paradigm, wherein each video was coded in relation to the previous video. As such, participants observed a series of short videos, each $2.67 \mathrm{sec}$ in duration, within which gaze direction was pseudorandomly varied. It could be that aSTS did not show sensitivity because of the relatively transient suppression design employed in the current study; a prior adaptation period may have been necessary. Further RS studies are required to test this hypothesis.

Other brain imaging studies that have not used RS designs have consistently implicated pSTS with processing different aspects of gaze direction. Some studies show that pSTS responds more to direct gaze (Pelphrey et al., 2004), averted gaze (Hoffman \& Haxby, 2000; Puce et al., 1998), is equivalent for direct and averted gaze (Wicker et al., 1998), and some show no response in STS (George, Driver, \& Dolan, 2001; Kawashima et al., 1999). These conflicting results may also account for the absence of STS sensitivity to gaze direction in the current study. Each trial involved an actor looking directly toward the participant and then averting gaze in one direction, left or right. Therefore, each trial involved direct and averted gaze. Consequent suppression analysis of gaze direction (RS for gazed location) involved comparing trials with a repeated averted gaze direction with trials with a novel averted gaze direction. But both conditions also involved direct gaze. If STS responded strongly to direct gaze, or even equivalently to direct and averted, then our RS analysis is less likely to have shown STS sensitivity. In other words, on trials with a repeated averted gaze direction, where we predicted suppressed STS activity, STS may have still responded strongly in response to the direct gaze component of the stimuli. We cannot disentangle this issue with the current experimental design. However, because the focus of the present study was not on gaze direction but rather on deictic gaze perception, it was important to first include direct gaze to engage participants' attention (Senju \& Hasegawa, 2005). Future studies could try to separate these issues further.

\section{Conclusion}

The capacity to link other people's eye gaze with objects in the environment is an essential feature of social cognition, but prior work has not identified brain regions sensitive to object identity during deictic gaze scenarios. We show that pIPS is specifically sensitive to the identity of an object gazed at by an observed individual, irrespective of the spatial location of that object. This result complements and extends previous studies of gaze perception, which have shown that STS encodes gaze direction, whereas posterior parietal cortex reorients spatial attention. During the perception of other people's gaze, pIPS is involved in object-centered attention shifts in addition to spatial attention shifts. In the future, more sophisticated neurocognitive models must be formulated that take into 
account the time course, interactions, and development of different components of the social brain (Nummenmaa \& Calder, 2009). One starting place would be to investigate how linking eye gaze with objects in the environment interacts with other social processes that are central to understanding other people's gaze, such as understanding communicative intent and mental state inference.

Reprint requests should be sent to Richard Ramsey, Faculté de Psychologie et des Sciences de l'Education, Université catholique de Louvain, B-1348 Louvain-la-Neuve, Belgium, or via e-mail: richard.ramsey@uclouvain.be.

\section{REFERENCES}

Allison, T., Puce, A., \& McCarthy, G. (2000). Social perception from visual cues: Role of the STS region. Trends in Cognitive Sciences, 4, 267-278.

Andersen, R. A., Brotchie, P. R., \& Mazzoni, P. (1992). Evidence for the lateral intraparietal area as the parietal eye field. Current Opinion in Neurobiology, 2, 840-846.

Beauchamp, M. S., Haxby, J. V., Jennings, J. E., \& DeYoe, E. A. (1999). An fMRI version of the Farnsworth-Munsell 100-Hue Test reveals multiple color-selective areas in human ventral occipitotemporal cortex. Cerebral Cortex, 9, 257-263.

Becchio, C., Bertone, C., \& Castiello, U. (2008). How the gaze of others influences object processing. Trends in Cognitive Sciences, 12, 254-258.

Birmingham, E., \& Kingstone, A. (2009). Human social attention. Annals of the New York Academv of Sciences, 1156, 118-140.

Bisley, J. W., \& Goldberg, M. E. (2010). Attention, intention, and priority in the parietal lobe. Annual Review of Neuroscience, 33, 1-21.

Bristow, D., Rees, G., \& Frith, C. D. (2007). Social interaction modifies neural response to gaze shifts. Social Cognitive and Affective Neuroscience, 2, 52-61.

Calder, A. J., Beaver, J. D., Winston, J. S., Dolan, R. J., Jenkins, R., Eger, E., et al. (2007). Separate coding of different gaze directions in the superior temporal sulcus and inferior parietal lobule. Current Biology, 17, 20-25.

Chao, L. L., Haxby, J. V., \& Martin, A. (1999). Attribute-based neural substrates in temporal cortex for perceiving and knowing about objects. Nature Neuroscience, 2, 913-919.

Chao, L. L., \& Martin, A. (1999). Cortical regions associated with perceiving, naming, and knowing about colors. Iournal of Cognitive Neuroscience, 11, 25-35.

Corbetta, M., Patel, G., \& Shulman, G. L. (2008). The reorienting system of the human brain: From environment to theory of mind. Neuron, 58, 306-324.

Corbetta, M., \& Shulman, G. L. (2002). Control of goal-directed and stimulus-driven attention in the brain. Nature Reviews Neuroscience, 3, 201-215.

Culham, J. C., Cavina-Pratesi, C., \& Singhal, A. (2006). The role of parietal cortex in visuomotor control: What have we learned from neuroimaging? Neuropsychologia. 44 , 2668-2684.

Deaner, R. O., \& Platt, M. L. (2003). Reflexive social attention in monkeys and humans. Current Biology, 13, 1609-1613.

Driver, J., Davis, G., Ricciardelli, P., Kidd, P., Maxwell, E., \& Baron-Cohen, S. (1999). Gaze perception triggers reflexive visuospatial orienting. Visual Cognition, 6, 509-540.

Duvernoy, H. M. (1999). The buman brain: Surface, blood supply, and three-dimensional sectional anatomy.

New York: Springer-Verlag-Wein.
Emery, N. J. (2000). The eyes have it: The neuroethology, function and evolution of social gaze. Neuroscience and Biobehavioral Reviews, 24, 581-604.

Emery, N. J., Lorincz, E. N., Perrett, D. I., Oram, M. W., \& Baker, C. I. (1997). Gaze following and joint attention in rhesus monkeys (Macaca mulatta). Journal of Comparative Psychology, 111, 286-293.

Friesen, C. K., \& Kingstone, A. (1998). The eyes have it! Reflexive orienting is triggered by nonpredictive gaze. Psvchonomic Bulletin \& Review, 5, 490-495.

Friston, K. J., Worsley, K. J., Frackowiak, R. S. J., Mazziotta, J. C., \& Evans, A. C. (1994). Assessing the significance of focal activations using their spatial extent. Human Brain Mapping, 1, 210-220.

George, N., Driver, J., \& Dolan, R. J. (2001). Seen gaze-direction modulates fusiform activity and its coupling with other brain areas during face processing. Neuroimage, 13, 1102-1112.

Gnadt, J., \& Andersen, R. (1988). Memory related motor planning activity in posterior parietal cortex of macaque. Experimental Brain Research, 70, 216-220.

Grafton, S. T. (2009). Embodied cognition and the simulation of action to understand others. Annals of the New York Academv of Sciences, 1156, 97-117.

Grèzes, J., \& Decety, J. (2001). Functional anatomy of execution, mental simulation, observation, and verb generation of actions: A meta-analysis. Human Brain Mapping, 12, 1-19.

Grill-Spector, K., Kushnir, T., Edelman, S., Avidan, G., Itzchak, Y., \& Malach, R. (1999). Differential processing of objects under various viewing conditions in the human lateral occipital complex. Neuron, 24, 187-203.

Grill-Spector, K., \& Malach, R. (2001). fMR-adaptation: A tool for studying the functional properties of human cortical neurons. Acta Psychologica (Amsterdam), 107, 293-321.

Grosbras, M.-H., Laird, A. R., \& Paus, T. (2005). Cortical regions involved in eye movements, shifts of attention, and gaze perception. Human Brain Matbing, 25, 140-154.

Hagler, D. J., Jr., Riecke, L., \& Sereno, M. I. (2007). Parietal and superior frontal visuospatial maps activated by pointing and saccades. Neuroimage, 35, 1562-1577.

Hamilton, A. F., \& Grafton, S. T. (2006). Goal representation in human anterior intraparietal sulcus. Journal of Neuroscience, 26, 1133-1137.

Hamilton, A. F., \& Grafton, S. T. (2007). The motor hierarchy: From kinematics to goals and intentions. In P. Haggard, Y. Rosetti, \& M. Kawato (Eds.), Sensorimotor foundations of higher cognition: Attention and performance XXII (pp. 381-408). Oxford, UK: Oxford University Press.

Haxby, J. V., Gobbini, M. I., Furey, M. L., Ishai, A., Schouten, J. L., \& Pietrini, P. (2001). Distributed and overlapping representations of faces and objects in ventral temporal cortex. Science, 293, 2425-2430.

Haxby, J. V., Ungerleider, L. G., Clark, V. P., Schouten, J. L., Hoffman, E. A., \& Martin, A. (1999). The effect of face inversion on activity in human neural systems for face and object perception. Neuron, 22, 189-199.

Hietanen, J. K., Nummenmaa, L., Nyman, M. J., Parkkola, R., \& Hämäläinen, H. (2006). Automatic attention orienting by social and symbolic cues activates different neural networks: An fMRI study. Neuroimage, 33, 406-413.

Hoffman, E. A., \& Haxby, J. V. (2000). Distinct representations of eye gaze and identity in the distributed human neural system for face perception. Nature Neuroscience, 3, 80-84.

Hooker, C. I., Paller, K. A., Gitelman, D. R., Parrish, T. B., Mesulam, M. M., \& Reber, P. J. (2003). Brain networks for analyzing eye gaze. Cognitive Brain Research, 17, 406-418.

Ishai, A., Ungerleider, L. G., Martin, A., \& Haxby, J. V. (2000). The representation of objects in the human occipital and 
temporal cortex. Lournal of Cognitive Neuroscience, 12, $35-51$.

Ishai, A., Ungerleider, L. G., Martin, A., Schouten, J. L., \& Haxby, J. V. (1999). Distributed representation of objects in the human ventral visual pathway. Proceedings of the National Academv of Sciences. U.S.A., 96, 9379-9384.

Itti, L., \& Koch, C. (2000). A saliency-based search mechanism for overt and covert shifts of visual attention. Vision Research, 40, 1489-1506.

Kampe, K. K. W., Frith, C. D., \& Frith, U. (2003). "Hey John": Signals conveying communicative intention toward the self activate brain regions associated with "mentalizing," regardless of modality. Journal of Neuroscience, 23, 5258-5263.

Kawashima, R., Sugiura, M., Kato, T., Nakamura, A., Hatano, K. Ito, K., et al. (1999). The human amygdala plays an important role in gaze monitoring: A PET study. Brain 122, 779-783.

Klein, J. T., Shepherd, S. V., \& Platt, M. L. (2009). Social attention and the brain. Current Biology, 19, R958-R962.

Konen, C. S., \& Kastner, S. (2008). Representation of eye movements and stimulus motion in topographically organized areas of human posterior parietal cortex. Iournal of Neuroscience, 28, 8361-8375.

Kuzmanovic, B., Georgescu, A. L., Eickhoff, S. B., Shah, N. J., Bente, G., Fink, G. R., et al. (2009). Duration matters: Dissociating neural correlates of detection and evaluation of social gaze. Neuroimage, 46, 1154-1163.

Langton, S. R. H., \& Bruce, V. (1999). Reflexive visual orienting in response to the social attention of others. Visual Cognition, 6, 541-567.

Levy, I., Schluppeck, D., Heeger, D. J., \& Glimcher, P. W. (2007). Specificity of human cortical areas for reaches and saccades. Journal of Neuroscience, 27, 4687-4696.

Martin, A. (2007). The representation of object concepts in the brain. Annual Review of Psychology, 58, 25-45.

Materna, S., Dicke, P. W., \& Thier, P. (2008). Dissociable roles of the superior temporal sulcus and the intraparietal sulcus in joint attention: A functional magnetic resonance imaging study. Journal of Cognitive Neuroscience, 20, 108-119.

Naccache, L., \& Dehaene, S. (2001). The priming method: Imaging unconscious repetition priming reveals an abstract representation of number in the parietal lobes. Cerebral Cortex, 11, 966-974

Nummenmaa, L., \& Calder, A. J. (2009). Neural mechanisms of social attention. Trends in Cognitive Sciences, 13, 135-143.

Nummenmaa, L., Passamonti, L., Rowe, J., Engell, A. D., \& Calder, A. J. (2010). Connectivity analysis reveals a cortical network for eye gaze perception. Cerebral Cortex, 20, 1780-1787.

Pelphrey, K. A., Singerman, J. D., Allison, T., \& McCarthy, G. (2003). Brain activation evoked by perception of gaze shifts: The influence of context. Neuropsychologia, 41, 156-170.

Pelphrey, K. A., Viola, R. J., \& McCarthy, G. (2004). When strangers pass. Psvchological Science, 15, 598-603.

Perrett, D. I., Smith, P. A. J., Potter, D. D., Mistlin, A. J., Head, A. S., Milner, A. D., et al. (1985). Visual cells in the temporal cortex sensitive to face view and gaze direction. Proceedings of the Royal Society of London, Series B, Biological Sciences, 223, 293-317.

Pierno, A. C., Becchio, C., Tubaldi, F., Turella, L., \& Castiello, U. (2008). Motor ontology in representing gaze-object relations. Neuroscience Letters, 430, 246-251.

Pierno, A. C., Becchio, C., Wall, M. B., Smith, A. T., Turella, L., \& Castiello, U. (2006). When gaze turns into grasp. Iournal of Cognitive Neuroscience, 18, 2130-2137.
Puce, A., Allison, T., Bentin, S., Gore, J. C., \& McCarthy, G. (1998). Temporal cortex activation in humans viewing eye and mouth movements. Journal of Neuroscience, 18, $2188-2199$

Ramsey, R., \& Hamilton, A. F. d. C. (2010a). Triangles have goals too: Understanding action representation in left aIPS. Neuropsychologia, 48, 2773-2776.

Ramsey, R., \& Hamilton, A. F. (2010b). Understanding actors and object-goals in the human brain. Neuroimage, 50, $1142-1147$.

Ramsey, R., \& Hamilton, A. F. (in press). How does your own knowledge influence the perception of other people's actions in the human brain? Social Cognitive and Affective Neuroscience.

Redcay, E., Dodell-Feder, D., Pearrow, M. J., Mavros, P. L., Kleiner, M., Gabrieli, J. D. E., et al. (2010). Live face-to-face interaction during fMRI: A new tool for social cognitive neuroscience. Neuroimage, 50, 1639-1647.

Sakata, H., Taira, M., Murata, A., \& Mine, S. (1995). Neural mechanisms of visual guidance of hand action in the parietal cortex of the monkey. Cerebral Cortex, 5 , 429-438.

Saxe, R. (2006). Uniquely human social cognition. Current Opinion in Neurobiologv, 16, 235-239.

Schilbach, L., Wilms, M., Eickhoff, S. B., Romanzetti, S., Tepest, R., Bente, G., et al. (2010). Minds made for sharing: Initiating joint attention recruits reward-related neurocircuitry. Dournal of Cognitive Neuroscience, 22, $2702-2715$.

Schilbach, L., Wohlschlaeger, A. M., Kraemer, N. C., Newen, A., Shah, N. J., Fink, G. R., et al. (2006). Being with virtual others: Neural correlates of social interaction.

Neuropsychologia, 44, 718-730.

Schluppeck, D., Glimcher, P., \& Heeger, D. J. (2005). Topographic organization for delayed saccades in human posterior parietal cortex. Journal of Neurophysiology, 94, 1372-1384.

Senju, A., \& Hasegawa, T. (2005). Direct gaze captures visuospatial attention. Visual Cognition, 12, 127-144

Sereno, M. I., Pitzalis, S., \& Martinez, A. (2001). Mapping of contralateral space in retinotopic coordinates by a parietal cortical area in humans. Science, 94, 1350-1354.

Shepherd, S. V. (2010). Following gaze: Gaze-following behavior as a window into social cognition. Frontiers in Integrative Neuroscience, 4, 1-13.

Shepherd, S. V., Klein, J. T., Deaner, R. O., \& Platt, M. L. (2009). Mirroring of attention by neurons in macaque parietal cortex. Proceedings of the National Academy of Sciences 106, 9489-9494.

Silver, M. A., \& Kastner, S. (2009). Topographic maps in human frontal and parietal cortex. Trends in Cognitive Sciences 13, 488-495.

Simmons, W. K., Ramjee, V., Beauchamp, M. S., McRae, K., Martin, A., \& Barsalou, L. W. (2007). A common neural substrate for perceiving and knowing about color. Neuropsvchologia, 45, 2802-2810.

Tunik, E., Rice, N. J., Hamilton, A., \& Grafton, S. T. (2007). Beyond grasping: Representation of action in human anterior intraparietal sulcus. Neuroimage, 36(Suppl. 2), T77-T86.

Wicker, B., Michel, F., Henaff, M. A., \& Decety, J. (1998). Brain regions involved in the perception of gaze: A PET study. Neuroimage, 8, 221-227.

Williams, J. H., Waiter, G. D., Perra, O., Perrett, D. I., \& Whiten, A. (2005). An fMRI study of joint attention experience. Neuroimage, 25, 133-140. 
This article has been cited by:

1. Ulrich J. Pfeiffer, Kai Vogeley, Leonhard Schilbach. 2013. From gaze cueing to dual eye-tracking: Novel approaches to investigate the neural correlates of gaze in social interaction. Neuroscience \& Biobehavioral Reviews . [CrossRef]

2. Elisabetta Mazzarella, Antonia Hamilton, Luigi Trojano, Bianca Mastromauro, Massimiliano Conson. 2012. Observation of another's action but not eye gaze triggers allocentric visual perspective. The Quarterly Journal of Experimental Psychology 65, 2447-2460. [CrossRef] 